\title{
De la internacionalización a la transnacionalización en América Latina
}

Las notas que siguen tienen como propósito el reflexionar teóricamente sobre las nuevas características del sistema internacional en el cual se desenvuelven los países latinoamericanos. Dado el carácter preliminar de éstas, las ideas serán presentadas esquemáticamente para ser con posterioridad ampliadas en un contexto analfticamente diversificado.

1) América Latina desde su constitución como región polfticamente autónoma, en términos relativos, ha sido caracterizada por la creciente internacionalización de sus principales procesos internos.

Los estados-naciones del área han estado permanentemente afectados e involucrados en situaciones de origen extracontinental o bien han producido algún tipo de hechos de tal relevancia y magnitud que han impactado procesos o actores internacionales.

La internacionalización de las relaciones exteriores no es, en consecuencia, un proceso nuevo en la región. Más bien se podría decir que es parte de la naturaleza y carácter de los países del área.

El proceso de internacionalización, por otra parte, ha estado presente en casi toda la evolución e historia occidental. Con posterioridad a la apertura de China al comercio e influencia europea, prácticamente ningún área del globo queda fuera de algún tipo de vínculo externo o internacional. El aislacionismo queda reducido a una ideología que en la mayoría de los casos es más retórica que efectiva.

La expansión de las diversas economías, especialmente las europeas en el siglo pasado, se ve igualmente acompañada con la difusión de una variedad de ideas, cosmovisiones, religiones, políticas $y$ valores. A la expansión del intercambio de mercancías también le corresponden procesos similares en el plano de las ideas-mercancras, de las ideas-políticas y de otra serie de conceptos y valores.

Igualmente, la región se ve afectada por decisiones externas, tal como el decreto de libre comercio inglés en 1842, que obliga a la apertura de las economías latinoamericanas en forma progresiva. De alguna manera este tipo de medidas externas implican una ex- 
Augusto Varas ./ De la internacionalización a la transnacionalización...

ternacionalización de las decisiones locales, las cuales deben adecuarse a las primeras.

En el plano de las relaciones políticas, la internacionalización de ellas ha adoptado la forma de alianzas, coaliciones o acuerdos entre estados, los cuales han sido parte constitutiva de la conducta internacional de éstos. En el caso latinoamericano se pueden mostrar no sólo las conferencias de cancilleres y sus acuerdos hemisféricos, sino logros subregionales como los Pactos de Mayo u otros de tan diversa naturaleza como el Pacto Andino.

En suma, el proceso de internacionalización de la vida económica, politica y social ha sido un vector en permanente y constante ampliación.

2) Cuando se observà la actual situación internacional es necesario reconocer que junto a este proceso de internacionalización se ha sumado una nueva realidad que lo supone o comprende $y$, en algunos casos, lo supera en complejidad y profundidad.

La centralización y ancentración de la toma de derisiones a nivel intermacional vendria a ser el rasgo esencial que definiria la naturaleza de los nuevos procesos que han sido analizados bajo la optica de la transnacionalización. De este modo se concentran las decisiones en ámbitos (colectivos o institucionales) cada vez más reducidos en cuanto a su número y variedad.

$\mathrm{Si}$ tomamos el ejemplo del incremento de las tasas de interés mundial, producto del presupuesto deficitario estadounidense, encontramos que en él se expresa el nivel de internacionalización al cual ha llegado la economía mundial. Sin embargo, por muchos efectos que éste tenga y por numerosas que sean las personas e instituciones y países afectados, difícilmente lo podrfamos concebir dentro del proceso de transnacionalización. Para que éste esté presente se deben cumplir dos requisitos previos. Por una parte, la fijación de objetivos comunes para el conjunto de actores involucrados $y / 0$ afectados por determinadas medidas $y$, por la otra, el acuerdo o pacto sobre las medidas, mecanismos o instrumentos a través de los cuales tales objetivos se alcanzarán. Sea un actor detérminado (empresa, partido o iglesia), sea un conjunto de ellos, el proceso de nuevo tipo que emerge con fuerza en los últimos años es la coordinación de éstos en una estrategia de maximización de sus potencialidades para alcanzar mayores niveles de logro en cada uno de sus campos especificos.

Esta nueva realidad debe ser diferenciada de la aspiración de determinados actores por alcanzar tales niveles de burocratización. Al contrario de la mera voluntad por lograr niveles superiores de maximización organizacional, el proceso de burocratización inherente a la planificación y programación transnacional es un recorrido que sólo se puede mostrar cuando en cada ámbito determinado los actores respectivos se acercan al tipo puro ideal de control total.y 
completo de la variedad del mercado o sistema politico internacional.

Para alcanzar este nivel superior de desarrollo, el proceso de transnacionalización de las diversas organizaciones debe transcurrir en un medio paradojalmente diversificado. En efecto, se podría establecer que el proceso de internacionalización tuvo como resultado un sistema internacional en el cual los acuerdos, alianzas o coaliciones se desarrollaron de tal forma que dieron la imagen, especialmente durante la guerra fría, que se tendía a una coordinación en gran escala de las actividades económicas, políticas o ideológicas. No obstante, el espejismo se destruye en la medida que tales acuerdos internacionales comienzan a ser erosionados por la emergencia, dentro del mismo sistema internacional, de organizaciones que se transnacionalizan efectivamente. Así, tienden a coexistir acuerdos o bloques con procesos emergentes de transnacionalización.

En la medida que avanza la transnacionalización, centralizándose y concentrándose la toma de decisiones, la unidad de la pluralidad se fragmenta a través de la emergencia en el seno de cada acuerdo internacional de actores con aspiraciones no sólo hegemónicas, sino excluyentes. La transnacionalización adquiere asi su rasgo centralizador más claro al establecer su existencia sobre la base de la exclusión de los distintos "mercados" del resto de los "competidores".

En síntesis, a diferencia del permanente curso de Ia internacionalización, la transnacionalización al concentrar y centralizar tomas de decisiones en mercados específicos, tiene el efecto inicial de fragmentar los respectivos sistemas.

3) Establecidas las relaciones entre ambos procesos, es necesario identificar el estado en el cual se encuentra el sistema internacional a este respecto.

Si bien es posible identificar un vigoroso proceso de transnacionalización a todo nivel (económico, político e ideológico-cultural) se debe reconocer el actual momento o fase como de transición de un sistema caracterizado por la plena internacionalización a otro tendiente a una más desarrollada transnacionalización. Todo ello en un marco de fragmentación de los diversos centros de poder tradicionales con el anteriormente mencionado efecto de diversificación del sistema internacional.

Es esta fase de transición la que le imprime carácter a la época actual en el campo internacional. Así, en la medida que el sistema se caracteriza por un doble y simultáneo proceso de descomposición/recomposición, serán las características de éstos las que definirán la naturaleza de los procesos internacionales actualmente observados.

En primer lugar, es posible caracterizar la transición como una fase de agudización de la competencia por el control de los diversos ámbitos internacionalizados a través de pocas unidades o acto- 
res que'se proyectan en la perspectiva de maximizar sus beneficios, De esta forma, la transnacionalización no excluye, en esta fase de transición, el agudo proceso de competencia y rivalidad entre los diversos actores que tienden a concentrar y centralizar en torno a sí cada uno de tales ámbitos. Por ello la transnacionalización se podría ver no como la eliminación ipso facto de la concurrencia en todos los planos, sino.comi una agudización de ella, especialmente en esta fase transitiva.

En segúndo lúgar, esta competencia se da en un contexto mencionado como de fragmentación del poder mundial. Fragmentación que se puede observar en todos y cada uno de los grarides ámbitos en los cuales hemos hecho referencia. En la medida que la internacionalización integró cada vez más elementos, su capacidad enrolvente en un primer momento se vio como ilimitada; sin embargo, en un segundo momento ella mostró que la puesta en escena simultáneamente de tal diversidad de actores llevaba ineluctablemente a retroalimentaciones, oposiciones y contradicciones que finalmente diluyeron los marcos de la internacionalización pre-existente. Un caso ilustrativo es la creciente internacionalización del socialismo soviético (ideológica, política, militar y económicamente), la que al llegar a su máximo proceso de inclusividad comienza a mostrar una fragmentación y. una emergencia de diversos polos ideológicos, po: líticos, militares y económicos que cuestionan la hegemonía de la URSs en ese sistema. Es a partir de este proceso que se agudiza la aspiración de dominación de algunos de sưs elementos tendiendo a concentrar y centralizar, al margen de mecanismos de acuerdos, coaliciones, o frentes, al resto de los actores en torno a un solo factor.

Una tercera característica sería la ampliación del espacio posible para la generación de la nueva competencia en esta fase de transnacionalización. En la medida que aumentan los elementos disponibles, se agudiza la concurrencia y se fragmenta el poder mundial en diversos respectos, el espacio o amplitud de la capacidad de acción de los entes transnacionalizados aumenta considerablemente. Al:romperse las barreras de las coaliciones y acuerdos, quedan disponibles espacios económicos, políticos e ideológicos para ser llenados por estos nuevos entes: altamente burocratizados, supranacionales, que se coordinan en la perspectiva de la maximización de sus beneficios.

En resumen, el momento actual debería comprenderse como de transición a un mayor énfasis en el término transnacionalizador del binomio descomposición/recomposición del sistema internacional.

4) Quisiéramos esquematizar aún más nuestro análisis formalizando el carácter desigual y combinado que adopta la escena internacional al ser conceptualizada como de transición, en torno a las características que hemos mencionado recién.

$\mathrm{Si}$ - categorizáramos los procesos observados a nivel internacional 
en torno a los ejes internacionalización/transnacionalización, e hiciéramos algo similar con los actores o portadores de cada uno de estos tipos o construidos, podríamos tener las siguientes combinaciones:

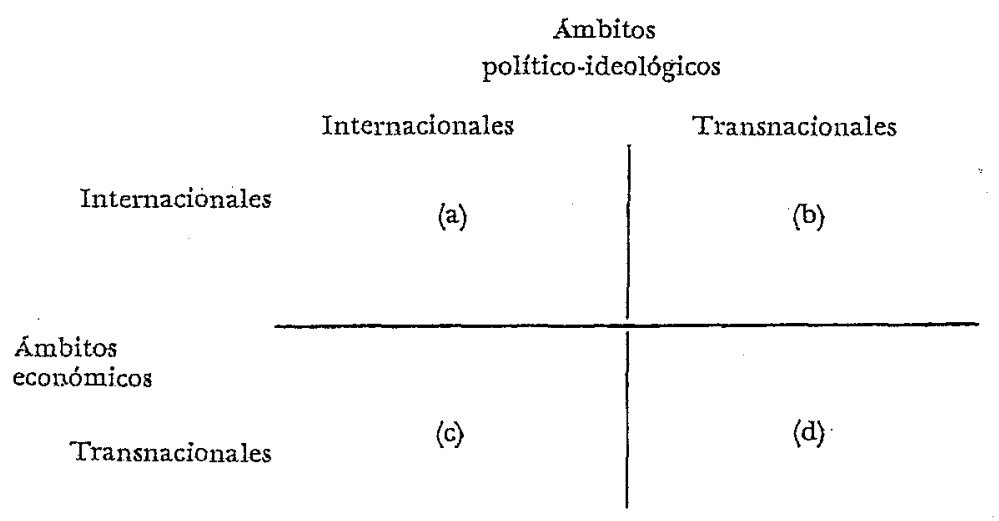

De las relaciones tipos que hemos establecido se pueden obtener cuatro categorizaciones generales o construidos tipológicos.

a) Internacionalización politico-econóniica: En éste se pueden agrupar todos aquellos procesos típicos de la fase de descomposición del sistema internacional que hemos caracterizado en los primeros puntos de nuestro análísis. Eligiendo arbitrariamente un ejemplo que tipificaría este grupo de procesos podríamos mencionar la actual internacionalización de las FF.AA. en América Latina. Así, roto el marco de coherencia y cohesión hemisférica, las FF.AA. de la región diversifican sus relaciones con otros cuerpos armados intra y extracontinentales, así como establecen relaciones económicas con un número cada vez más amplio de proveedores. En ninguno de estos casos podríamos hablar de un proceso de transnacionalización de los institutos castrenses, a no ser que forzáramos el argumento en el plano de lo ideológico en forma unilateral. Aún así las Fr.AA. difícilmente se podrían analizar desde la perspectiva de otro tipo de proceso a nivel global.

b) Transnacionalización político-ideológica $e$ internacionalización económica: En este grupo de procesos podríamos identificar todos aquelios que se refieren al despliegue internacional de fuerzas ideológico-políticas que aspiran a ejercer un control burocrático de nuevo tipo sobre el conjunto de los actores internacionales. Su vínculo económico es establecido fundamentalmente en el plano de los diversos flujos e intercambios observados a nivel mundial, más qúe concentrar su acción en el plano de un solo proceso de intercambio económico. Un ejemplo destacado de este tipo es la nueva activi- 
dad desarrollada por la Ira Internacional Socialista en América Latina, la cual fija prioridades de desempeño en subregiones o países especificos, a partir de una política de maximización de potencialidades a escala global. Igualmente, los mecanismos y las formas de accionar locales tienden a homogenizarse. Las últimas tensiones en su seno, producto de oposiciones internas, muestran la tendencia a cerrar las fronteras institucionales, profundizar el consenso y actuar como ente altamente burocratizado en la política latinoamericana. Sus vínculos políticos, si bien podrían privilegiar determinados flujos económicos no están afectados por éstos.

c) Internacionalización politica y transnacionalización económica: En éste se agrupan procesos que tienen su origen fundamentalmente en la definición conscientemente diseñada y controlada de maximización de beneficios a escala mundial. Ejemplo destacado de este tipo de procesos es la unificación a nivel mundial de los diversos capitales financieros de origen nacional en una estrategia global de maximización de utilidades. Sus altos niveles de burocratización están operacionalizados en el accionar de los diversos centros financieros mundiales, la eliminación a través de ellos de las limitaciones temporo-espaciales y el control creciente no sólo del mercado financiero sino de otros mercados que se ligan cada vez más en forma estrecha a ellos. Desde el punto de vista ideológico, si bien se despliega una iniciativa tendiente a diversificar las relaciones en cada región, subregión y país, a partir de determinadas fuentes de producción ideológica (neo-conservadurismo y monetarismo neo-liberal) no existe un nivel de burocratización (centralización y concentración) equivalente al observado en el plano económico. Más bien el signo es la diversificación de esta función intelectual a nivel global.

d) Transnacionalización politico-ideológica y económica: En este tipo encontramos aquellos procesos que tienden a acercarse al tipo puro ideal de transnacionalización que mencionáramos anteriormente. Los niveles de concentración y centralización de la toma de decisiones son superiores, la maximización de beneficios en cada uno de los ámbitos que corresponda es buscada a nivel global, la operación es realizada a través de entes descentralizados pero articulados a una matriz que fija planes, programas, y metas, y evalúa resultados corrigiendo desempeños. En este tipo de procesos cabe una realidad característica de la época actual, tales son los conglomerados de medios de comunicación de masas que al mismo tiempo de maximizar utilidades económicas a escala mundial, homogenizan los contenidos, valores y concepciones de mundo que son simultáneamente mercancias/valores. Esta nueva realidad de potenciar renta con influencia y persuasión, ha comenzado a inundar, a través de sus canales propios, a prácticamente la totalidad del mundo occidental. Aquí los niveles de burocratización son superiores; sin 
Augusto Taras / De la internacionalización a la transnacionalización ...

cipales conglomerados multinacionales. No obstante, en. el plano político e ideológico, Brasil muestra los efectos de la internacionaIización en su seno al no expresar mecánicamente ninguna influencia determinante en la dirección del estado. Más bien este es un ámbito de resolución de conflictos por la hegemonía, pero en el cual siempre la fracción dominante mantiene su capacidad de direçción sobre el conjunto:

Aun cuando es difícil establecer casos representativos de un tipo puro ideal, sería posible, al mantener las distancias pertinentes, mencionar a Jamaica como un caso que expresa algunas de las características del tipo (d). Con posterioridad a la elección de Manley, este país se vio fuertemente afectado por las orientaciones y política estadounidense en el Caribe. $\mathrm{La}$ desestabilización política también se expresó en la presión de conglomerados multinacionales que controlan gxan parte de su riqueza básica. Así, Seaga de alguna manera expresa el restablecimiento del equilibrio hegemónico entre la política económica de transnacionales productivas y las aspiraciones políticas del actor dominante en esa escena, tales son los EE. UU. En estas condiciones, el estado pierde crecientemente su papel de dirección social y pasa a representar un mecanismo de ges* tión de intereses externalizados. El vaciamiento de lo nacional llega a sus niveles más altos.

6) Al analizar el sistema internacional de los ochenta a partir. de una perspectiva de este tipo es posible ubicar analíticamente el problema de la interdependencia y el desarrollo nacional.

Si se acepta que el sistema internacional se encuentra inmerso en un proceso de transición entre la descomposición de las agrupaciones características de una fase de internacionalización, hacia el polo de la recomposición transnacional, se puede entender cómo y por qué los ámbitos tradicionales del sistema de relaciones multigubernamentales comienzan a perder capacidad de orientación y obligan toriedad. El deterioro existente, más que expresar la crisis del derecho internacional, muestra la falta de capacidad política de tales organismos para aplicar una normativa sobre un conjunto de países que adoptan lógicas de funcionamiento tan diversas como.Ias posibles combinaciones que se pueden generar a partir de la matriz simple antes descrita.

Desde este punto de vista, la interdependencia se pone en el plano de los diversos ámbitos (actores y procesos) donde se construye y desarrolla el proceso de concentración y centralización de la toma de decisiones. Es en este campo donde diversos elementos, supra o-subordinados, pueden alcanzar una relación de complementariedad que se expresa en una interdependencia de sus procesos particulares.. La unificación de los grandes conglomerados internacionales en el.último año es un buen ejemplo de tal situación en los EE. UU. Si bien la unificación. de conglomerados se . hace en tor- 
no a un polo dominante, las relaciones técnicas entre ellos son de complementaridad. Igual cosa sucede en la extensión de las actividades de empresas multinacionales a áreas tradicionalmente no privilegiadas en su inversión. Tal es el caso de la incursión de los grandes conglomerados de los EE. UU. en el sector energético, por razones obvias.

Otro tipo de complementariedades se pueden apreciar en esquemas interdependientes como el COMECON $\mathrm{u}$ otras experiencias de integración regional o subregional. Igual cosa sucede con la interdependencia de la integración realizada por multinacionales productivas al vincular diversos procesos nacionales coordinados supranacionalmente.

La interdependencia se establece fundamentalmente cuando se genera una complementariedad técnica, una división del trabajo, en el marco de la acción de un ente dominante en torno al cual se ordena (supra y subordinaciones) el conjunto de los elementos partícipes de esta relación "orgánica". De esta forma, Ia interdepen. dencia se ubica en el centro de los fenómenos típicos de la transnacionalización en los cuales la división técnica del trabajo es consecuencia del dominio irrestricto de un actor sobre el resto.

Algo diferente ocurre en el marco de la internacionalización. Aquí los vínculos que se establecen no modifican esencialmente el tipo de relación, la cual puede suspenderse o no $\sin$ poner en peligro de manera inmediata la existencia de sus partes. Tal situación se puede observar en las relaciones de países exportadores de petróleo/consumidores de él, las que se alteran y logran perder su carácter o grado de complementariedad cuando los países industrializados sustituyen energía, bajan la producción y la opep debe verse obligada a bajar los precios. Así, la interdependencia se muestra como estrecho vínculo y no como complementariedad.

Extremando el argumento se podría decir que la interdependencia se establece cuando las partes técnicamente integradas quedan sometidas al poder de un elemento subordinado cuando éste, como lo mencionaba un economista, hace uso de su capacidad de "colapso final".

7) Finalmente, establecido el problema de la interdependencia al interior del proceso transnacionalizador, es preciso destacar que desde el punto de vista del estado nacional, y en consecuencia, de la población afectada por estos procesos, la interdependencia transnacionalizadora somete a fuertes presiones a los respectivos estados para convertirlos en correa de transmisión de los procesos globales.

En este contexto, el control democrático del estado se plantea como un requisito funcional en la medida que su fortaleza dependerá de los soportes sociales que éste tenga. En esa misma medida tal estado podrá mantener su nivel de autonomía frente a las tendencias transnacionalizadoras al establecer, en vez de relaciones interdepen- 
Augusto Varas / De la internacionalización a la transnacionalización...

dientes, vínculos exteriores que no comprometan directa y mecánicamente sus decisiones internas.

Los términos de relación entre estados aún no transnacionalizados y el contexto internacional global pasa, en consecuecia, por un doble proceso. Por una parte, por la capacidad de ejecutar políticas que cuenten con respaldos masivos y por ende puedan aspirar a la estabilidad. Por la otra, por la integración política en coaliciones amplias de iguales que desarrollen políticas comunes para enfrentar las presiones provenientes del marco internacional transnacionalizado.

La capacidad selectiva de los estados nacionales para incorporar los beneficios del entorno internacional sólo podrá ser maximizada cuando tales relaciones de poder a nivel global queden efectivamente balanceadas. 